\title{
Magnetism and orbitally driven spin-singlet states in Ru oxides: A muon-spin rotation study
}

\author{
S. J. Blundell,* T. Lancaster, P. J. Baker, and W. Hayes \\ Clarendon Laboratory, University of Oxford, Parks Road, Oxford OX1 3PU, United Kingdom \\ F. L. Pratt \\ ISIS Muon Facility, ISIS, Chilton, Oxon. OX11 OQX, United Kingdom \\ T. Atake \\ Tokyo Institute of Technology, 4259 Nagatsuta-cho, Midori-ku, Yokohama 226-8503, Japan
}

D. S. Rana ${ }^{\dagger}$ and S. K. Malik

Tata Institute of Fundamental Research, Colaba, Mumbai 400 005, India

(Received 21 August 2007; revised manuscript received 28 January 2008; published 20 March 2008)

\begin{abstract}
We have used zero-field muon-spin rotation to study the local magnetic properties of $\mathrm{SrRuO}_{3}, \mathrm{Y}_{2} \mathrm{Ru}_{2} \mathrm{O}_{7}$, and $\mathrm{La}_{2} \mathrm{RuO}_{5}$. While $\mathrm{SrRuO}_{3}$ and $\mathrm{Y}_{2} \mathrm{Ru}_{2} \mathrm{O}_{7}$ give rise to coherent precession signals, in $\mathrm{La}_{2} \mathrm{RuO}_{5}$ we find no signature of long range magnetic order below the structural transition, but observe relaxation consistent with that expected due to a dimerization into spin-singlet states. In $\mathrm{Y}_{2} \mathrm{Ru}_{2} \mathrm{O}_{7}$ we observe two precession signals with different temperature dependences, consistent with the nature of the noncollinear ordering being temperature dependent.
\end{abstract}

DOI: 10.1103/PhysRevB.77.094424

PACS number(s): 76.75.+i, 75.25.+z, 75.30.Et

\section{INTRODUCTION}

The rich variety of behavior exhibited by transition metal oxides arises because of the interplay between spin, charge, and orbital degrees of freedom and the variety of lattice architectures which are available. ${ }^{1}$ Spin ordering, both ferromagnetic and antiferromagnetic, and spin frustration can be observed in various phases, as can charge order. ${ }^{2,3}$ In addition, the orbital degeneracy in the transition metal ions can be lifted by an interaction with the lattice ${ }^{4}$ and this can lead to orbital ordering, the best-known case of which is the cooperative Jahn-Teller distortion in $\mathrm{LaMnO}_{3}{ }^{5}$ Furthermore, the Coulomb interaction between electrons is important for understanding metal-insulator transitions and properties such as high-temperature superconductors and colossal magnetoresistance observed in certain oxides, and the orbital degrees of freedom can play a role in these phenomena. ${ }^{6}$

Though most work has been performed on first-row transition metal oxides, ruthenium-based oxides have also proved to be very attractive to study in this context. The ruthenium ion contains $4 d$ orbitals, whose extended nature might be expected to lead to a weakened tendency to electron localization compared with $3 d$ analogs for which electron-electron correlations are known to be significant. Nevertheless, ruthenium-based oxides can display itinerant or localized behavior depending on chemical environment, resulting in a rich variety of physical properties. ${ }^{7}$ For example, $\mathrm{Sr}_{2} \mathrm{RuO}_{4}$ is a $p$-wave superconductor, ${ }^{8} \mathrm{SrRuO}_{3}$ is a ferromagnetic metal, ${ }^{9} \mathrm{La}_{2} \mathrm{RuO}_{5}$ is a semiconductor, ${ }^{10}$ and $\mathrm{Y}_{2} \mathrm{Ru}_{2} \mathrm{O}_{7}$ is a magnetic insulator. ${ }^{11}$ These systems all possess $\mathrm{Ru}^{4+}$ ions, which each have four $4 d$ electrons in a low-spin configuration $\left(t_{2 g}^{4}\right)$, and all comprise three-dimensional networks of corner-sharing $\mathrm{RuO}_{6}$ octahedra. Nevertheless, differences in crystal structure alter the $4 d$ bandwidth $W$ and, when this is very narrow (and becomes comparable to the on-site correlation $U$ ), correlation-induced electron localiza- tion of the $4 d$ electrons can occur. ${ }^{12}$ Moreover, understanding the role of the relevant orbital and spin degrees of freedom is crucial for comprehending the rich variety of behavior found in these strongly correlated oxides, ${ }^{1,13}$ and this is particularly relevant for $\mathrm{La}_{2} \mathrm{RuO}_{5}$ (Ref. 10), which displays an unusual structural transition accompanied by novel orbital physics. ${ }^{14,15}$

To shed light on these issues, we have used muon-spin rotation $(\mu \mathrm{SR})$ to probe the magnetism in three compounds containing $\mathrm{Ru}^{4+}$ ions but which are constructed by assembling $\mathrm{RuO}_{6}$ octahedra in different architectures: (i) $\mathrm{SrRuO}_{3}$, a three-dimensional distorted pseudocubic perovskite of the $\mathrm{GdFeO}_{3}$ type ${ }^{22}$ in which there is a zig-zag tilting of the $\mathrm{RuO}_{6}$ octahedra [see Fig. 1(a)]; (ii) $\mathrm{Y}_{2} \mathrm{Ru}_{2} \mathrm{O}_{7}$, a cubic $(F d \overline{3} m)$ pyrochlore structure in which $\mathrm{Ru}^{4+}$ ions form a threedimensional network of corner-sharing tetrahedra [see Fig. 1(b)]; (iii) $\mathrm{La}_{2} \mathrm{RuO}_{5}$, which contains double (110) perovskite planes in which each $\mathrm{RuO}_{6}$ octahedron has corner-sharing

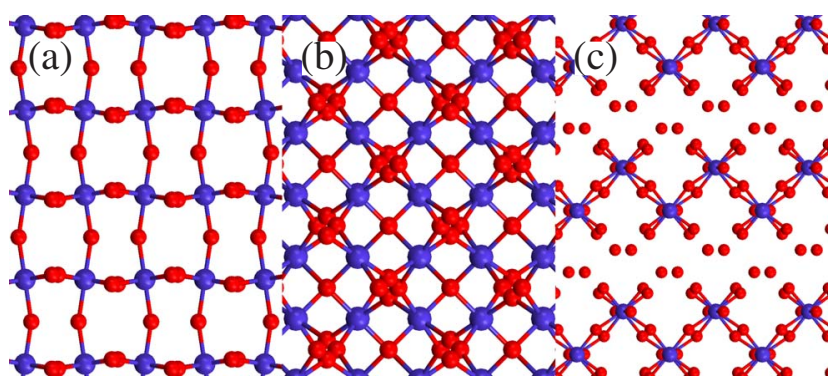

FIG. 1. (Color online) The crystal structure of (a) $\mathrm{SrRuO}_{3}$ (viewed down the $b$ axis, with $a$ horizontal and $c$ vertical), (b) $\mathrm{Y}_{2} \mathrm{Ru}_{2} \mathrm{O}_{7}$ (viewed down one of the cubic axes), and (c) $\mathrm{La}_{2} \mathrm{RuO}_{5}$ (viewed down the $c$ axis, with $a$ horizontal and $b$ vertical). The Ru atoms are shown in blue and the oxygen atoms in red. The Sr, Y, and La atoms are omitted for clarity. 
connections with four neighboring $\mathrm{RuO}_{6}$ octahedra [see Fig. $1(\mathrm{c})]$. In each case, we find that the $\mu \mathrm{SR}$ data are able to identify and characterize the different ground states.

\section{EXPERIMENTAL}

Our $\mu$ SR experiments were carried out using the GPS instrument at the Paul Scherrer Institute (PSI) in Switzerland and the ARGUS spectrometer at the ISIS Pulsed Muon Facility. Spin polarized positive muons $\left(\mu^{+}\right.$, momentum $28 \mathrm{MeV} / c$ ) were implanted into our samples. The muons stop quickly (in $<10^{-9} \mathrm{~s}$ ), without significant loss of spin polarization. The observed quantity is then the time evolution of the muon-spin polarization $P_{z}(t)$ obtained by studying the angular distribution of emitted decay positrons. ${ }^{23}$ The positrons are detected by using scintillation counters placed in front of and behind the sample. We record the number of positrons detected by forward $\left(N_{f}\right)$ and backward $\left(N_{b}\right)$ counters as a function of time and calculate the asymmetry function $G_{z}(t)$ using $G_{z}(t)=\left[N_{f}(t)-\alpha_{\exp } N_{b}(t)\right] /\left[N_{f}(t)\right.$ $\left.+\alpha_{\text {exp }} N_{b}(t)\right]$, where $\alpha_{\text {exp }}$ is an experimental calibration constant and differs from unity due to nonuniform detector efficiency, and $G_{z}(t) \propto P_{z}(t)$. The muon-spin precesses around a local magnetic field $B$, with a frequency $\nu=\left(\gamma_{\mu} / 2 \pi\right)|B|$, where $\gamma_{\mu} / 2 \pi=135.5 \mathrm{MHz} \mathrm{T}^{-1}$. The local magnetic field $B$ is a function of the muon-site $\mathbf{r}_{\mu}$ and can be written as

$$
B^{\alpha}\left(\mathbf{r}_{\mu}\right)=\sum_{i} D_{i}^{\alpha \beta}\left(\mathbf{r}_{\mu}\right) m_{i}^{\beta},
$$

a sum over the magnetic ions in the crystal; the magnetic moment of the $i$ th ion is $\mathbf{m}_{i}$ and is located at $\mathbf{r}_{i}$. In Eq. (1) $D_{i}^{\alpha \beta}\left(\mathbf{r}_{\mu}\right)$ is the dipolar tensor given by

$$
D_{i}^{\alpha \beta}\left(\mathbf{r}_{\mu}\right)=\frac{\mu_{0}}{4 \pi R_{i}^{3}}\left(\frac{3 R_{i}^{\alpha} R_{i}^{\beta}}{R_{i}^{2}}-\delta^{\alpha \beta}\right),
$$

where $\mathbf{R}_{i} \equiv\left(R_{i}^{x}, R_{i}^{y}, R_{i}^{z}\right)=\mathbf{r}_{\mu}-\mathbf{r}_{i}$. The behavior of this tensor is dominated by the arrangement of the nearest-neighbor magnetic ions and leads to a nonzero local magnetic field for almost all possible muon sites, even in an antiferromagnetically ordered system. ${ }^{16}$ Thus muon-spin precession can usually be observed in an antiferromagnet in zero applied magnetic field, even though there is no net magnetization (see, e.g., Ref. 17). In a polycrystalline sample, the muon polarization can be fitted to $P_{z}(t)=\frac{1}{3} e^{-\lambda_{\|}} t+\frac{2}{3} e^{-\lambda t} \cos \left(\gamma_{\mu}|B| t\right)$ for the case of a single muon site, where $\lambda_{\|}$and $\lambda$ are relaxation rates; this expression reflects that, on average, two-thirds of the incident muon polarization will lie perpendicular to the local field. This expression can be generalized for multiple sites and can be corrected for the possibility of a fraction of muons which implant outside the sample and thus contribute to a background signal.

Muons implanted in oxide materials tend to find sites close to oxygen anions and form an analog of the hydroxide ion $\left(\mathrm{OH}^{-}\right)$via the reaction $\mu^{+}+\mathrm{O}^{2-} \rightarrow \mathrm{OMu}^{-}$, resulting in a diamagnetic state which is sensitive to local fields. ${ }^{18}$ It is also possible to form neutral muonium $\left(\mathrm{Mu}^{0}=\mu^{+} e^{-}\right)$; this is a paramagnetic center, so that the muon experiences a hyperfine field which adds to or subtracts from the applied field. ${ }^{18,19}$ Even for the diamagnetic $\mathrm{OMu}^{-}$site, it is possible in a magnetic oxide to have a contribution to the local field from the supertransfer hyperfine interaction. ${ }^{20}$ This results from overlap between the Mu $1 s$ orbital and the oxygen $2 p$ orbital, which in turn transfers an electron to an unoccupied $\mathrm{Ru} 4 d$ orbital; this supertransfer transfer field would add to the dipolar field in Eq. (1), though the dipolar field is the dominant term. ${ }^{21}$

\section{RESULTS}

\section{A. $\mathrm{SrRuO}_{3}:$ A ferromagnetic metal}

First, we present data on polycrystalline $\mathrm{SrRuO}_{3}$ (our sample was prepared as described in Ref. 22). The $163^{\circ} \mathrm{Ru}-$ $\mathrm{O}-\mathrm{Ru}$ bond angle is close to $180^{\circ}$ and might therefore lead one to expect antiferromagnetic interactions (due to the Goodenough-Kanamori rules) but, unusually for a perovskite, $\mathrm{SrRuO}_{3}$ is found to be a metallic ferromagnet below $T_{C}=160 \mathrm{~K}$ (see, e.g., Ref. 9). The $t_{2 g}^{4}$ electronic state predicts $S=1$ and a moment of $2 \mu_{B}$, but experiments yield $0.8-1.6 \mu_{B}$, pointing to band (rather than atomic) magnetism. ${ }^{24}$ One also expects a half-metallic ground state $\left(t_{2 g}^{3} \uparrow, t_{2 g}^{1} \downarrow\right)$ with the majority spin subband insulating and the minority spin subband conducting. The electronic heat capacity ${ }^{9,25}$ shows that the effective mass is enhanced $\left(m^{*}\right.$ $\approx 3 m_{e}$ ), consistent with $\mathrm{SrRuO}_{3}$ being on the border of a Mott-Hubbard transition, ${ }^{26,27}$ though this interpretation has been questioned. ${ }^{28}$ Hybridization of the ruthenium $t_{2 g}$ orbitals and the oxygen $2 p$ orbitals $^{29}$ is important in this system, and it has been pointed out that the nature of the hybridization is controlled by the orbital orientation of the $t_{2 g}$ electrons in such a way as to be able to explain non-Fermi liquidlike features in the optical spectra. ${ }^{30}$ It has also been predicted that the on-site Coulomb interactions can drive orbital order on the minority spin band. ${ }^{31}$ These results all demonstrate the subtle interplay between orbital physics, Jahn-Teller distortions, and the on-site correlation.

The $\mu$ SR data are shown in Fig. 2 and demonstrate that very weak spin relaxation just above $T_{C}$ [the $165 \mathrm{~K}$ trace in Fig. 2(a)] changes abruptly to a damped coherent oscillation below $T_{C}$, the frequency of which increases rapidly on cooling [see Figs. 2(a) and 2(b); note the different horizontal scales of these two figures]. At $5 \mathrm{~K}$ [see Fig. 2(b)], the dominant precession frequency corresponds to a quasistatic local field at the muon site of $0.25 \mathrm{~T}$. The oscillations are heavily damped (probably due to the field distribution resulting from domain structure ${ }^{32}$ ), though not as strongly as those observed in Ref. 33, pointing to a well-defined magnetic environment of the muon in our samples. These data are consistent with the development of ferromagnetic order below $T_{C}$. Reference 33 demonstrates how this local field collapses as $\mathrm{Sr}$ is progressively replaced by $\mathrm{Ca}$, a process which reduces the $\mathrm{Ru}-$ $\mathrm{O}-\mathrm{Ru}$ angle, narrows the bandwidth, and increases the effect of the electron correlations. In fact, it is believed that there is a transfer of spectral weight from the quasiparticle peak at $E_{F}$ to the Hubbard bands as one goes from $\mathrm{SrRuO}_{3}$ to $\mathrm{CaRuO}_{3}$ and finally, to the insulating pyrochlore $\mathrm{Y}_{2} \mathrm{Ru}_{2} \mathrm{O}_{7}$, which we consider in the next section. 

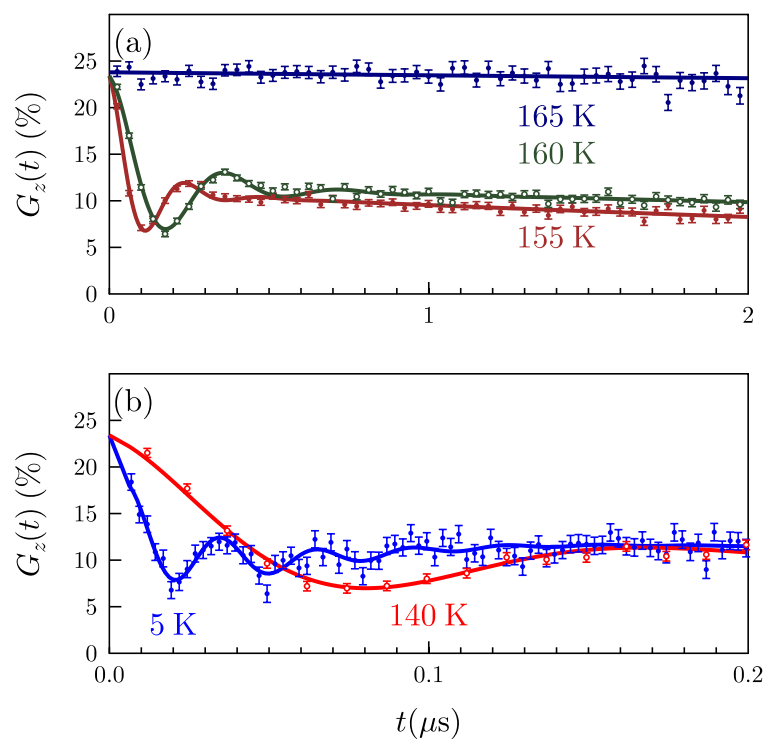

FIG. 2. (Color online) Raw $\mu$ SR data for $\mathrm{SrRuO}_{3}$ at selected temperatures viewed in the time range (a) $0-2 \mu \mathrm{s}$ and (b) $0-0.2 \mu \mathrm{s}$.

\section{B. $\mathbf{Y}_{2} \mathbf{R u}_{2} \mathbf{O}_{7}:$ A magnetic insulator}

The small $\mathrm{Y}^{3+}$ cation leads to a smaller $\mathrm{Ru}-\mathrm{O}-\mathrm{Ru}$ angle $\left(128^{\circ}\right)$ in $\mathrm{Y}_{2} \mathrm{Ru}_{2} \mathrm{O}_{7}$, which reduces the bandwidth, ${ }^{34}$ leading to insulating behavior because of correlation-induced electron localization. ${ }^{12,35,36} \mathrm{Y}_{2} \mathrm{Ru}_{2} \mathrm{O}_{7}$ exhibits a peak in the susceptibility and heat capacity at $T_{N}=76 \mathrm{~K},{ }^{37}$ though the magnitude of the Curie-Weiss temperature $\left(\Theta_{W} \approx-1100 \mathrm{~K}\right)$ is much larger than $T_{N}$, which is indicative of the presence of strong frustration. ${ }^{37,38}$ In fact, the transition has been described as spin-glass-like, ${ }^{37,39}$ though far-infrared spectra show strong antiferromagnetic correlations (which can be ascribed to a significant spin-phonon coupling ${ }^{40}$ ) and these have been studied in detail using inelastic neutron scattering. ${ }^{41}$ Raw $\mu$ SR data on a polycrystalline sample of $\mathrm{Y}_{2} \mathrm{Ru}_{2} \mathrm{O}_{7}$ are shown in Fig. 3(a) and demonstrate that a coherent muon precession signal develops below $T_{N}$.

These data confirm that $\mathrm{Y}_{2} \mathrm{Ru}_{2} \mathrm{O}_{7}$ undergoes antiferromagnetic ordering below $T_{N}$ (in agreement with Ref. 42 and the correlations observed in Ref. 40); a glassy or short-rangeordered ground state would give rise to a much more heavily damped signal and, in fact, short-range-order has been ruled out on the basis of optical measurements. ${ }^{35}$ The amplitude of the oscillations accounts for all the muons stopped in the sample and allows us to conclude that there is long-range ordering throughout the bulk. The band gap of $\mathrm{Y}_{2} \mathrm{Ru}_{2} \mathrm{O}_{7}$ is about $1.5 \mathrm{eV}$, similar to that of $\mathrm{Ag}_{2} \mathrm{O}$, though while the latter compound shows a missing fraction of muons corresponding to the formation of neutral muonium, ${ }^{18}$ the muons in $\mathrm{Y}_{2} \mathrm{Ru}_{2} \mathrm{O}_{7}$ remain diamagnetic.

Neutron diffraction ${ }^{39}$ and ${ }^{99} \mathrm{Ru}$ Mössbauer ${ }^{43}$ data provide evidence that the magnetic order below $T_{N}$ is not collinear, consistent with the expected geometric frustration in this compound. The beating pattern visible in the $45 \mathrm{~K}$ data plotted in Fig. 3(a) is the result of two separate precession signals with similar frequencies, showing that there are two (a)

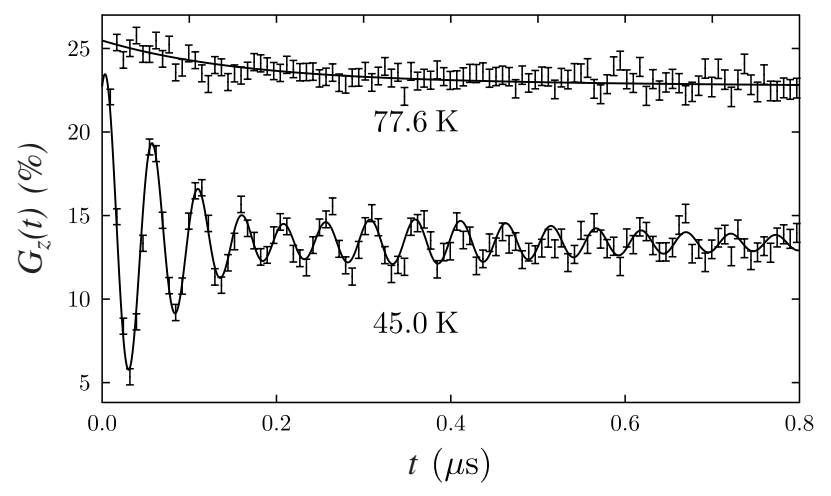

(b)

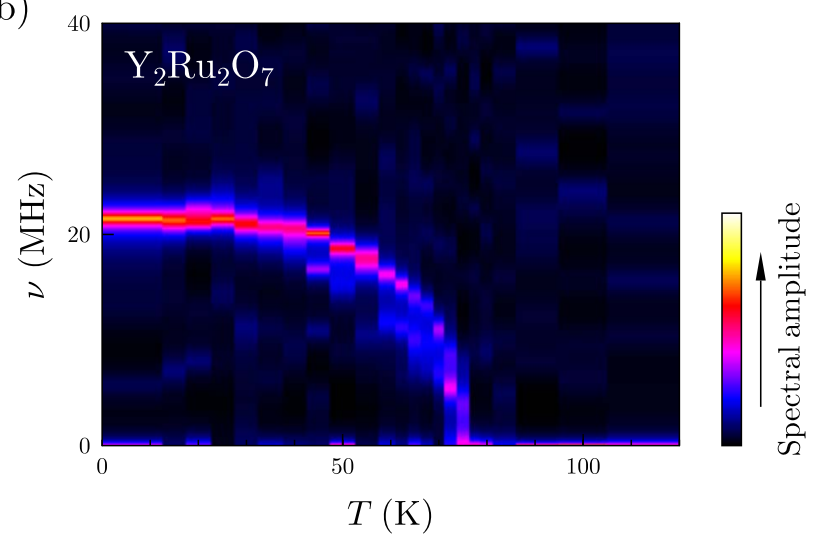

FIG. 3. (Color online) (a) Raw $\mu$ SR data for $\mathrm{Y}_{2} \mathrm{Ru}_{2} \mathrm{O}_{7}$ at two temperatures. Two distinct precession frequencies are observed below $T_{N}=67 \mathrm{~K}$. The solid line is a time-domain fit using parameters in Figs. 4(b) and 4(d). (b) Temperature evolution of the frequency spectrum extracted from maximum entropy analysis. This shows two closely spaced modes (indistinguishable at low temperature, but separating at higher temperature); the less distinct, lowerfrequency mode decreases faster with increasing temperature.

magnetically inequivalent muon sites, which is consistent with the noncollinear nature of the magnetic order. Maximum entropy analysis was used to decompose the signal as a function of temperature and the results are shown in Fig. 3(b). The two frequencies in the spectrum are difficult to distinguish at all temperatures, but it is clear that they follow markedly different temperature dependences, with the lowerfrequency falling to zero faster than the higher-frequency on warming.

These trends are easier to extract using fitting of the muon spectrum in the time domain. The data were fitted to an expression involving two oscillating components (each exponentially relaxing) and a very weakly relaxing background. The amplitudes of the two components were fixed over the measured temperature range, the amplitude of the lowerfrequency component being a factor of $\approx 2.2$ higher than the higher-frequency component.

The temperature dependence of the fitted frequencies of the two precession signals is plotted in Fig. 4(b). This shows, in agreement with Fig. 3(b), that both frequencies collapse to zero at $T_{N}$, the temperature at which there is a peak in the 


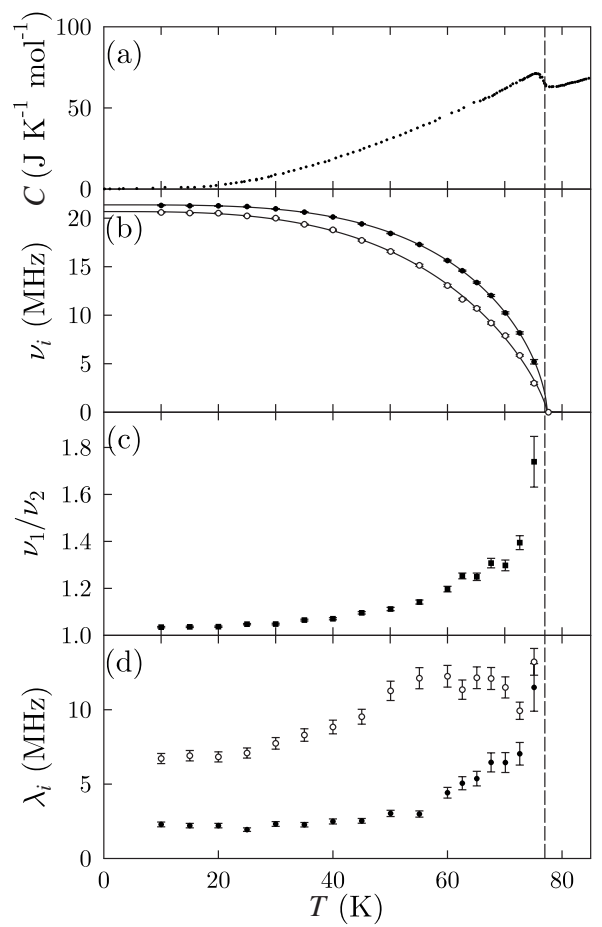

FIG. 4. Data for $\mathrm{Y}_{2} \mathrm{Ru}_{2} \mathrm{O}_{7}$. (a) Heat capacity data (after Ref. 11). (b) Fitted precession frequencies $\nu_{1}$ (closed circles) and $\nu_{2}$ (open circles) (the solid lines are guides to the eye), (c) the ratio of the two frequencies, and (d) the corresponding relaxation rates.

heat capacity [Fig. 4(a)]. However, the two components follow a different temperature dependence, with the higherfrequency component collapsing to zero more slowly than the lower-frequency component [see Fig. 4(c)]. The damping rate of the lower-frequency component is also substantially larger than that of the higher-frequency component [Fig. 4(d)]. This is consistent with the maximum entropy spectra in Fig. 3(b), which show that the lower-frequency component is broader, even though it accounts for more than double the spectral weight of the higher-frequency component.

Our results suggest that the relative orientation of the magnetic moments in the unit cell may be temperature dependent, leading to the differing temperature dependence of the local field at the two magnetically inequivalent muon sites. This can be due to a continuous reorientation of the spins on different magnetic sublattices which might be expected if the precise details of the ordered state depend on the fine balance of competing exchange interactions, at least one of which is temperature dependent. It is also possible that the signal ascribed to a lower-frequency site may actually be from a number of magnetically inequivalent sites with very slightly different local fields, thus contributing to the broad feature in the maximum entropy spectra.

\section{C. $\mathrm{La}_{2} \mathrm{RuO}_{5}$ : A semiconductor}

We now consider $\mathrm{La}_{2} \mathrm{RuO}_{5}$, which undergoes a semiconductor-semiconductor transition at $T_{s} \approx 160 \mathrm{~K}$ accompanied by a sharp drop in the magnetic susceptibility. ${ }^{10,44}$ The symmetry is monoclinic $\left(P 2_{1} / c\right)$ above the transition, but triclinic $(P \overline{1})$ below it. ${ }^{10,45}$ This behavior was initially interpreted in terms of an orbital ordering in which $t_{2 g \uparrow}^{3} t_{2 g \downarrow}^{1}$ $S=1$ moments in the high-temperature state transform into $t_{2 g \uparrow}^{2} t_{2 g \downarrow \downarrow}^{2} S=0$ moments ${ }^{10}$ (specifically a $d_{x y}^{2} d_{x z}^{2} d_{y z}^{0}$ configuration) in the low-temperature state. However, another possibility is orbital-driven singlet pair formation ${ }^{46}$ in which the $S=1$ moments present at high temperature remain at low temperature, but in the form of singlet dimers stabilized by an orbital ordering. In this picture, the high-temperature $t_{2 g}$ manifold consists of a superposition of $d_{x y}^{2} d_{x z}^{1} d_{y z}^{1}, d_{x y}^{1} d_{x z}^{2} d_{y z}^{1}$, and $d_{x y}^{1} d_{x z}^{1} d_{y z}^{2}$ configurations, and the structural transition and consequent lowering of symmetry drive the ion into a $d_{x y}^{1} d_{x z}^{2} d_{y z}^{1}$ configuration at low temperature; pairs of ions with such a configuration then dimerize. This viewpoint is supported by density functional calculations within the local density approximation. ${ }^{14}$ Further confirmation is provided by x-ray absorption spectroscopy measurements ${ }^{15}$ which show that at $T<T_{s}$ the $\mathrm{Ru}^{4+}$ ions remain in an $S=1$ state.

Muon experiments on a sample of polycrystalline $\mathrm{La}_{2} \mathrm{RuO}_{5}$ (prepared as described in Ref. 44) were carried out first at PSI, and these established that there was no signal corresponding to a quasistatic local field at any temperature in the range 5-300 K. A more detailed study of the temperature dependence of the relaxation was carried out on the ARGUS spectrometer at ISIS. The results of these latter $\mu$ SR experiments are shown in Fig. 5 and provide additional evidence that there is no magnetic transition to long-range order in this material. The raw experimental data are shown in Fig. 5(a) for selected temperatures [a small temperatureindependent background term for these data has been subtracted and the data are plotted in the form $\left.P_{z}(t)\right]$. It is clear from these data, which are quite different from those obtained for $\mathrm{SrRuO}_{3}$ and $\mathrm{Y}_{2} \mathrm{Ru}_{2} \mathrm{O}_{7}$, that no long-range ordering takes place.

The data over the whole studied temperature range ${ }^{47}$ were fitted to the "stretched-exponential" function $P_{z}(t)=e^{-(\lambda t)^{\beta}}$ and the fitted parameters are plotted in Figs. 5(c) and 5(d). We find that $\lambda$ is approximately independent of temperature apart from a small increase which sets in at low temperatures. The shape parameter $\beta$ approaches 2 at high temperature, but falls to $\approx 0.5$ at low temperature. We note that no sharp change in $\lambda$ or $\beta$ is observed as the sample is lowered through $T_{s}$ [although there is a clear peak in the heat capacity, see Fig. 5(b)]. The semiconducting gap in this compound is known to be very small $(\approx 0.2 \mathrm{eV})$ (Ref. 10), and so in comparison with other oxides with very small gaps ${ }^{18,19}$ we expect diamagnetic muon sites.

We can understand in general terms that exponential relaxation $(\beta=1)$ will result if the fluctuations in the local field at the muon site $B\left(\mathbf{r}_{\mu}\right)$ are characterized by a single correlation time $\tau$, and if the fluctuations are in the muon time window. The local field at the muon site will be dominated by the dipolar field due to Ru electronic moments. In the fast-fluctuation limit, ${ }^{48}$ the relaxation rate $\lambda$ $=2 \gamma_{\mu}^{2}\left\langle\left(\Delta B\left(\mathbf{r}_{\mu}\right)\right)^{2}\right\rangle \tau\left[\right.$ where $\left.\Delta B\left(\mathbf{r}_{\mu}\right)=B\left(\mathbf{r}_{\mu}\right)-\left\langle B\left(\mathbf{r}_{\mu}\right)\right\rangle\right]$. Hence, if the Ru moments are fluctuating very rapidly (as they will in the high-temperature paramagnetic state), $\tau$ is very short and $\lambda \rightarrow 0$ (motional-narrowing limit), and one may observe only the remaining relaxation which results from the dipolar 

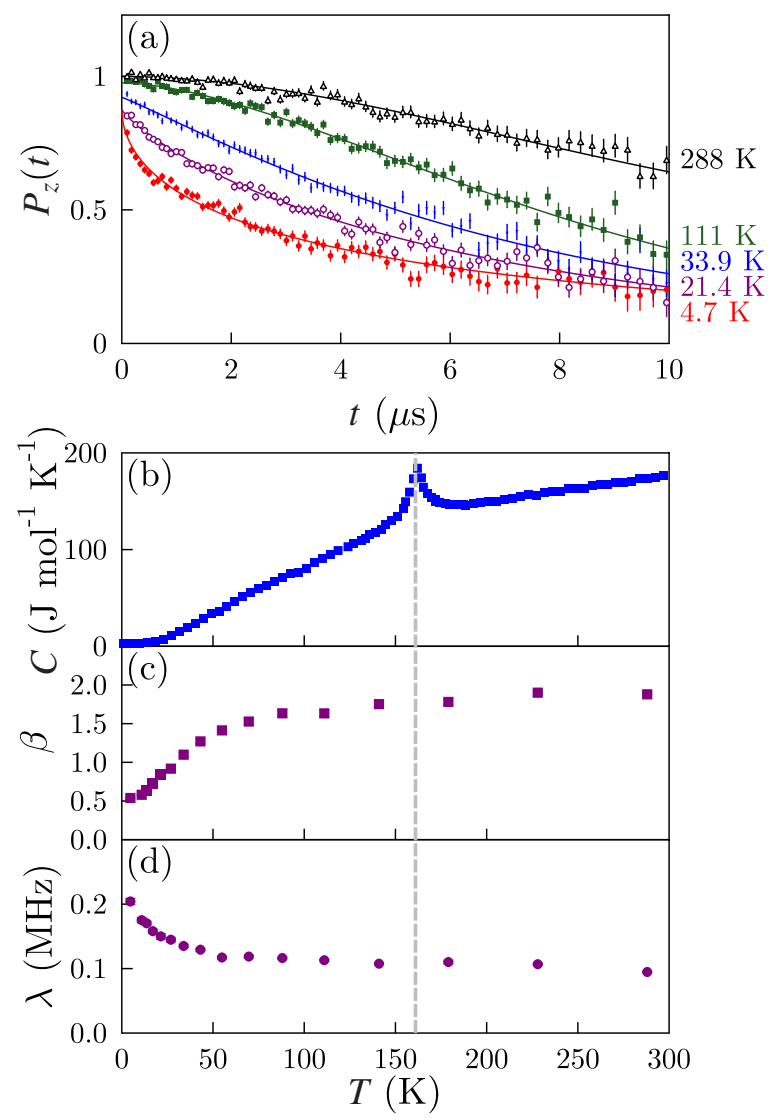

FIG. 5. (Color online) (a) Raw $\mu$ SR data for $\mathrm{La}_{2} \mathrm{RuO}_{5}$ for selected temperatures. (b) Heat capacity data (after Ref. 44). (c) The fitted values of the shape parameter $\beta$. (d) The fitted values of the relaxation rate $\lambda$.

field produced by the static nuclear moments $(\beta=2)$. This is what we find at high temperatures. In the fully dimerized state, all the electronic moments should disappear since all molecules are now $S=0$ and the relaxation will again be due only to nuclear moments $(\beta=2)$, although this is not observed. Instead, the presence of a few undimerized pairs and/or defects may lead to a very dilute distribution of moments which fluctuate with a unique relaxation time $\tau$; this leads to a "root-exponential" $\left(\beta=\frac{1}{2}\right)$ relaxation, ${ }^{49}$ which is what we find at low temperature.
Between the low- and high-temperature limits, we observe a smooth crossover from $\beta=0.5$ to $\beta=2$ relaxation. A similar smooth evolution of muon relaxation rate through a dimerization transition is observed in related systems. ${ }^{50,51}$ Since our data are strongly reminiscent of those in systems which undergo a spin-Peierls transition (in agreement with Ref. 15), it seems likely that the $S=1$ moments remain below the transition to the low-temperature state but their dynamics remains fast on the muon time scale; further cooling to low temperature induces a crossover to a behavior dominated by defect spins. ${ }^{50}$

\section{CONCLUSION}

In summary, our $\mu \mathrm{SR}$ experiments have demonstrated the variety of behavior observable in $\mathrm{Ru}$-based oxides with different crystal architectures. Both $\mathrm{SrRuO}_{3}$ and $\mathrm{Y}_{2} \mathrm{Ru}_{2} \mathrm{O}_{7}$ give rise to coherent precession signals, demonstrating long-range magnetic order below the ordering transitions. $\mathrm{SrRuO}_{3}$ is known to be a ferromagnet, but the transition in $\mathrm{Y}_{2} \mathrm{Ru}_{2} \mathrm{O}_{7}$ has been described as "spin-glass-like" and our data effectively rule out this interpretation. Two precession frequencies are observed in the pyrochlore $\mathrm{Y}_{2} \mathrm{Ru}_{2} \mathrm{O}_{7}$, resulting from two magnetically inequivalent sites, but their temperature dependences differ markedly. This is consistent with the nature of the noncollinear ordering being temperature dependent, possibly resulting from the fact that the magnetic order is a rather subtle "trade off" between competing interactions. In $\mathrm{La}_{2} \mathrm{RuO}_{5}$ we find no signature of long-range magnetic order below the structural transition, but observe relaxation consistent with that expected due to a dimerization into spin-singlet states.

\section{ACKNOWLEDGMENTS}

The sample of $\mathrm{SrRuO}_{3}$ was prepared by P. P. C. Frampton and P. D. Battle of the Inorganic Chemistry Laboratory, Oxford. We thank EPSRC (UK) for financial support. This research project has also been supported by the European Commission under the Sixth Framework Programme through the Key Action: Strengthening the European Research Area, Research Infrastructures, Contract No. RII3-CT-2003505925. T.L. acknowledges support from the Royal Commission for the Exhibition of 1851. Part of this work was performed at the Swiss Muon Source, Paul Scherrer Institute, Villigen, Switzerland. The authors thank Alex Amato and Robert Scheuermann for technical assistance at PSI. *s.blundell@physics.ox.ac.uk

Present address: Institute of Laser Engineering, Osaka University, 2-6 Yamadaoka, Suita 565-0871, Osaka, Japan.

Present address: International Center for Condensed Matter Physics (ICCMP), University of Brasilia, Brasilia, Brazil.

${ }^{1}$ M. Imada, A. Fujimori, and Y. Tokura, Rev. Mod. Phys. 70, 1039 (1998).

${ }^{2}$ P. G. Radaelli, D. E. Cox, M. Marezio, and S.-W. Cheong, Phys. Rev. B 55, 3015 (1997).
${ }^{3}$ E. Wawrzynska, R. Coldea, E. M. Wheeler, I. I. Mazin, M. D. Johannes, T. Sorgel, M. Jansen, R. M. Ibberson, and P. G. Radaelli, Phys. Rev. Lett. 99, 157204 (2007); T. Lancaster, S. J. Blundell, P. J. Baker, M. L. Brooks, W. Hayes, F. L. Pratt, R. Coldea, T. Sörgel, and M. Jansen, ibid. 100, 017206 (2008).

${ }^{4}$ D. I. Khomskii and M. V. Mostovoy, J. Phys. A 36, 9197 (2003).

${ }^{5}$ J. B. Goodenough, Phys. Rev. 100, 564 (1955).

${ }^{6}$ Y. Tokura and N. Nagaosa, Science 288, 462 (2000).

${ }^{7}$ R. J. Cava, Dalton Trans. 2004, 2979. 
${ }^{8}$ A. P. Mackenzie and Y. Maeno, Rev. Mod. Phys. 75, 657 (2003).

${ }^{9}$ P. B. Allen, H. Berger, O. Chauvet, L. Forro, T. Jarlborg, A. Junod, B. Revaz, and G. Santi, Phys. Rev. B 53, 4393 (1996).

${ }^{10}$ P. Khalifah, R. Osborn, Q. Huang, H. W. Zandbergen, R. Jin, Y. Liu, D. Mandrus, and R. J. Cava, Science 297, 2237 (2002).

${ }^{11}$ S. Yoshii and M. Sato, J. Phys. Soc. Jpn. 68, 3034 (1999), and references therein.

${ }^{12}$ P. A. Cox, R. G. Egdell, J. B. Goodenough, A. Hamnett, and C. C. Naish, J. Phys. C 16, 6221 (1983).

${ }^{13}$ D. I. Khomskii, Phys. Scr. 72, CC8 (2005).

${ }^{14}$ V. Eyert, S. G. Ebbinghaus, and T. Kopp, Phys. Rev. Lett. 96, 256401 (2006).

${ }^{15} \mathrm{Hua}$ Wu, Z. Hu, T. Burnus, J. D. Denlinger, P. G. Khalifah, D. G. Mandrus, L.-Y. Jang, H. H. Hsieh, A. Tanaka, K. S. Liang, J. W. Allen, R. J. Cava, D. I. Khomskii, and L. H. Tjeng, Phys. Rev. Lett. 96, 256402 (2006).

${ }^{16}$ S. J. Blundell, Philos. Trans. R. Soc. London, Ser. A 357, 2923 (1999).

${ }^{17}$ S. J. Blundell, C. A. Steer, F. L. Pratt, I. M. Marshall, W. Hayes, and R. C. C. Ward, Phys. Rev. B 67, 224411 (2003).

${ }^{18}$ S. F. J. Cox, J. S. Lord, S. P. Cottrell, J. M. Gil, H. V. Alberto, A. Keren, D. Prabhakaran, R. Scheuermann, and A. Stoykov, J. Phys.: Condens. Matter 18, 1061 (2006).

${ }^{19}$ S. F. J. Cox, J. L. Gavartin, J. S. Lord, S. P. Cottrell, J. M. Gil, H. V. Alberto, J. Piroto Duarte, R. C. Vilão, N. Ayres de Campos, D. J. Keeble, E. A. Davis, M. Charlton, and D. P. van der Werf, J. Phys.: Condens. Matter 18, 1079 (2006).

${ }^{20}$ C. Boekema, R. L. Lichti, V. A. M. Brabers, A. B. Denison, D. W. Cooke, R. H. Heffner, R. L. Hutson, M. Leon, and M. E. Schillaci, Phys. Rev. B 31, 1233 (1985).

${ }^{21}$ C. Boekema, R. L. Lichti, and K. J. Rüegg, Phys. Rev. B 30, 6766 (1984).

${ }^{22}$ C. W. Jones, P. D. Battle, P. Lightfoot, and W. T. A. Harrison, Acta Crystallogr., Sect. C: Cryst. Struct. Commun. C45, 365 (1989).

${ }^{23}$ S. J. Blundell, Contemp. Phys. 40, 175 (1999).

${ }^{24}$ J. M. Longo, P. M. Raccah, and J. B. Goodenough, J. Appl. Phys. 39, 1327 (1968).

${ }^{25}$ G. Cao, S. McCall, Z. X. Zhou, C. S. Alexander, J. E. Crow, R. P. Guertin, and C. H. Mielke, Phys. Rev. B 63, 144427 (2001).

${ }^{26}$ J. S. Ahn, J. Bak, H. S. Choi, T. W. Noh, J. E. Han, Yunkyu Bang, J. H. Cho, and Q. X. Jia, Phys. Rev. Lett. 82, 5321 (1999).

${ }^{27}$ P. Kostic, Y. Okada, N. C. Collins, Z. Schlesinger, J. W. Reiner, L. Klein, A. Kapitulnik, T. H. Geballe, and M. R. Beasley, Phys. Rev. Lett. 81, 2498 (1998).

${ }^{28}$ K. Maiti and R. S. Singh, Phys. Rev. B 71, 161102(R) (2005).

${ }^{29}$ N. Hiraoka, M. Itou, A. Deb, Y. Sakurai, Y. Kakutani, A. Koizumi, N. Sakai, S. Uzuhara, S. Miyaki, H. Koizumi, K. Makoshi, N. Kikugawa, and Y. Maeno, Phys. Rev. B 70, 054420 (2004).

${ }^{30}$ M. S. Laad and E. Müller-Hartmann, Phys. Rev. Lett. 87, 246402 (2001).

${ }^{31}$ H.-T. Jeng, S.-H. Lin, and C.-S. Hsue, Phys. Rev. Lett. 97, 067002 (2006).
${ }^{32}$ L. Klein, Y. Kats, A. F. Marshall, J. W. Reiner, T. H. Geballe, M. R. Beasley, and A. Kapitulnik, Phys. Rev. Lett. 84, 6090 (2000).

${ }^{33}$ Y. J. Uemura, T. Goko, I. M. Gat-Malureanu, J. P. Carlo, P. L. Russo, A. T. Savici, A. Aczel, G. J. MacDougall, J. A. Rodriguez, G. M. Luke, S. R. Dunsiger, A. McCollam, J. Arai, Ch. Pfleiderer, P. Böni, K. Yoshimura, E. Baggio-Saitovitch, M. B. Fontes, J. Larrea, Y. V. Sushko, and J. Sereni, Nat. Phys. 3, 29 (2007).

${ }^{34}$ K.-S. Lee, D.-K. Seo, and M.-H. Whangbo, J. Solid State Chem. 131, 405 (1997).

${ }^{35}$ J. S. Lee, Y. S. Lee, T. W. Noh, K. Char, Jonghyurk Park, S.-J. Oh, J.-H. Park, C. B. Eom, T. Takeda, and R. Kanno, Phys. Rev. B 64, 245107 (2001).

${ }^{36}$ J. Park, K. H. Kim, H.-J. Noh, S.-J. Oh, J.-H. Park, H.-J. Lin, and C.-T. Chen, Phys. Rev. B 69, 165120 (2004).

${ }^{37}$ S. Yoshii and M. Sato, J. Phys. Soc. Jpn. 68, 3034 (1999).

${ }^{38}$ H. Sakai, H. Kato, S. Kambe, R. E. Walstedt, H. Ohno, M. Kato, K. Yoshimura, and H. Matsuhata, Phys. Rev. B 66, 100509(R) (2002).

${ }^{39}$ M. Ito, Y. Yasui, M. Kanada, H. Harashina, S. Yoshii, K. Murata, M. Sato, H. Okumura, and K. Kakurai, J. Phys. Soc. Jpn. 69, 888 (2000).

${ }^{40}$ J. S. Lee, T. W. Noh, J. S. Bae, I.-S. Yang, T. Takeda, and R. Kanno, Phys. Rev. B 69, 214428 (2004).

${ }^{41}$ J. van Duijn, N. Hur, J. W. Taylor, Y. Qiu, Q. Z. Huang, S.-W. Cheong, C. Broholm, and T. G. Perring, Phys. Rev. B 77, 020405(R) (2008).

${ }^{42}$ M. Tachibana, H. Kawaji, and T. Atake, Phys. Rev. B 71, 060402(R) (2005).

${ }^{43}$ R. Kmieć, Z. Światkowska, J. Gurgul, M. Rams, A. Zarzycki, and K. Tomala, Phys. Rev. B 74, 104425 (2006).

${ }^{44}$ S. K. Malik, D. C. Kundaliya, and R. D. Kale, Solid State Commun. 135, 166 (2005).

${ }^{45}$ S. G. Ebbinghaus, Acta Crystallogr., Sect. C: Cryst. Struct. Commun. 61, i96 (2005).

${ }^{46}$ D. I. Khomskii and T. Mizokawa, Phys. Rev. Lett. 94, 156402 (2005).

${ }^{47}$ Below $100 \mathrm{~K}$, there is a very small reduction in the initial asymmetry which was included in the fitting by allowing $P_{z}(0)$ to reduce slightly from unity. In addition, for the highest temperature run, there is some evidence of the onset of muon diffusion.

${ }^{48}$ N. Bloembergen, E. M. Purcell, and R. V. Pound, Phys. Rev. 73, 679 (1948).

${ }^{49}$ Y. J. Uemura, T. Yamazaki, D. R. Harshman, M. Senba, and E. J. Ansaldo, Phys. Rev. B 31, 546 (1985).

${ }^{50}$ S. J. Blundell, F. L. Pratt, P. A. Pattenden, M. Kurmoo, K. H. Chow, T. Jestädt and W. Hayes, J. Phys.: Condens. Matter 9, L119 (1997); B. W. Lovett, S. J. Blundell, F. L. Pratt, T. Jestadt, W. Hayes, S. Tagaki, and M. Kurmoo, Phys. Rev. B 61, 12241 (2000).

${ }^{51}$ P. J. Baker, S. J. Blundell, F. L. Pratt, T. Lancaster, M. L. Brooks, W. Hayes, M. Isobe, Y. Ueda, M. Hoinkis, M. Sing, M. Klemm, S. Horn, and R. Claessen, Phys. Rev. B 75, 094404 (2007). 